\title{
Cochlear and Auditory Brainstem Implants in the Management of Acoustic Neuroma and Bilateral Acoustic Neurofibromatosis
}

\author{
Neil L. Davis*§, B.A., Jamie M. Rappaport ${ }^{\S}$, M.D., \\ James C. MacDougall $\$$, Ph.D.
}

\section{INTRODUCTION}

Though both are methods for restoring partial hearing to the deaf, cochlear and auditory brainstem implants are clearly appropriate for separate patient populations. The fundamental factor which differentiates patients in each population is the integrity and utility of the cochlear nerve. In terms of acoustic neuroma, most patients are not candidates for cochlear implantation due to the damage done to the cochlear nerve by the lesion and its surgical excision, as well as the fact that the vast majority of acoustic neuroma patients have useful hearing in the non-tumor ear. In general, the only acoustic neuroma patients who would be cochlear implant candidates are those who have coincident loss of hearing in the non-tumor ear (which is associated with a functionally intact cochleovestibular nerve) and they would require an implant on this side. In rare cases, patients with a functionally intact cochlear nerve after tumor removal may be considered for subsequent cochlear implantation. Most acoustic neuroma patients who require implants, however, receive the auditory brainstem implant (ABI), and these are almost always individuals with neurofibromatosis type-2 (NF-2) who characteristically develop bilateral acoustic tumors. This review considers the current prosthetic technology

\footnotetext{
* To whom correspondence should be addressed

$\S$ Department of Otolaryngology, Jewish General Hospital, 3755 Cote-Ste-Catherine Road, Montreal, Quebec, Canada, H3T 1E2

$\dagger$ Department of Otolaryngology, McGill University, Montreal, Quebec, Canada

\# Department of Psychology, McGill University, 1205 Dr. Penfield Ave., Montreal, Quebec, Canada, H3A 1B1
}

used for the rehabilitation of patients who have lost hearing as a result of tumors of the eighth cranial (cochleovestibular) nerve.

\section{ACOUSTIC NEUROMAS AND THEIR SURGICAL MANAGEMENT}

Acoustic neuromas are benign, slow-growing schwannomas of the cochleovestibular nerve. There are several possible presenting symptoms, including tinnitus, facial numbness, mild disequilibrium, and others (1). Most commonly, a patient will present with gradual sensorineural hearing loss in the affected ear. It has been noted, however, that in up to $12 \%$ of cases, the patient may experience a more sudden loss of hearing (2).

While usually occurring without other pathology, acoustic neuromas can also be symptomatic of the autosomal dominant disorder known as bilateral acoustic neurofibromatosis (also referred to as NF-2). NF-2, with its associated bilateral cochlear neuromas, is significantly rarer than unilateral acoustic neuromas. Acoustic neuromas are diagnosed at the rate of one per 100000 individuals each year in the United States, and only $5 \%$ of these individuals actually exhibit the bilateral tumors associated with NF-2 at the time of diagnosis (3). Most patients with NF-2 begin showing symptoms of the disorder at a young age and acoustic neuromas found in adults are more likely to be sporadic (4). In addition to bilateral vestibular schwannomas, NF-2 patients are predisposed to various schwann cell tumors, including cranial and spinal meningiomas, nerve root neurofibromas and gliomas of the brainstem and spinal cord (4). 
Table 1. Summary of cases in which the cochlear implant and the auditory brainstem implant are likely to be considered for use in acoustic neuroma patients.

Cochlear Implant

Can be used in patients with sensorineural hearing loss on the side contralateral to the acoustic tumor who have a functionally intact cochleovestibular nerve on that side. Patient must also have an adequate surviving spiral ganglion cell population on the side of implantation, if it is to be successful. Often of no help on the affected side, since the cochleovestibular nerve is usually functionally compromised during the surgical removal of the lesion. However, in rare cases when the nerve is left functionally intact but the cochlea is left damaged, the cochlear implant has been used successfully.

Auditory Brainstem Implant

Is currently used only in patients with NF-2 and is always implanted simultaneously with tumor removal (usually during excision of the patient's second tumor). It is useful in patients who have had both cochleovestibular nerves sacrificed, since this implant stimulates the cochlear nuclear complex in the brainstem directly.

Once a diagnosis of acoustic neuroma has been made, there are several effective management strategies which exist. The choice of management strategy will depend on multiple factors, including the size of the lesion, the overall health of the patient and, since the choice of surgical approach is based on whether hearing preservation is being considered, the amount of useful hearing which exists in both the affected and the unaffected ear at the time of diagnosis. There are three main surgical approaches to acoustic tumor removal (5). In the translabyrinthine approach, a mastoidectomy is performed and drilling then proceeds through the labyrinthe to expose the tumor within the internal auditory canal and cerebello-pontine angle (posterior fossa). As such, the lesion is approached through the auditory apparatus and, as a result, complete deafness always ensues. At this point, it is only via the translabyrinthine approach that the patient is implanted (with either ABI or cochlear implants) simultaneously with excision of the tumor (5). The tumor can also be removed via the suboccipital (or retrosigmoid) approach, by which hearing can potentially be salvaged, especially if the lesion is relatively small. Another approach which is routinely used is that via the middle fossa, which can only be used for tumors which are quite small; this approach also offers the possibility of hearing preservation (5).

Radiosurgery (a form of radiation therapy) is another management strategy which has become available in recent years. While attractive due to its non-invasive nature, it attempts merely to halt tumor growth and, as such, does not remove the lesion. It has been shown to be effective in most patients, but the long-term risks are not yet known and it has been speculated that tumor irradiation causes fibrosis in neighboring cranial nerves (6), thereby making subsequent microsurgical tumor removal more difficult, if necessary.

Finally, an observational approach is taken if a patient is elderly enough and the lesion is small enough; in these cases, it is assumed that the patient's life expectancy is shorter than the amount of time it will take for the tumor to pose a real threat (7).

\section{COCHLEAR IMPLANT}

First introduced in the 1960s, the cochlear implant has become a tool which is increasingly used to provide auditory stimulation to deafened individuals. In normal hearing, sound travels along the ear canal and vibrates the tympanic membrane (eardrum). The ossicular chain (malleus, incus, and stapes) conduct this vibration to the inner ear. Fluid waves in the cochlea stimulate the thousands of hearing (hair) cells of the inner ear. Movement of these hair cells generates an electrical current in the auditory nerve, which is then transmitted to the cochlear nuclei and further connections within the brainstem toward the auditory cortex of the brain.

Cochlear implants are presently considered in patients with various forms of sensorineural deafness (deafness due to abnormalities of the cochlea, auditory nerve, or central auditory pathways; this should be differentiated from hearing loss due to mechanical/conductive abnormalities of the ossicular chain or tympanic membrane, which can potentially be repaired using traditional otologic surgery techniques). Potential cochlear implant candidates must meet certain diagnostic criteria. These include a profound absence of residual hearing and a demonstrated inability to benefit from the amplification which would be provided by a conventional hearing aid device (Table 1) (8).

Cochlear implants are designed to bypass the hair cells of the inner ear and provide stimulation of the auditory nerve. There are several different cochlear implants currently available. All systems consist of a microphone and a signal processor which are worn outside the body like a hearing aid (Figure 1). A signal coupler (secured in the skin behind the ear) receives the stimulus, converts it to an electrical signal, and transmits this information to an electrode array that is implanted within the cochlea. These electrical signals stimulate the auditory nerve fibers, and this 




Figure 1. Schematic diagram depicting the components and anatomic location of the cochlear and auditory brainstem implants. Arrows indicate the direction of signal transmission along each system towards the brain. The microphone [1] picks up sound and sends it to the signal processor [2]. The processor selects particular characteristics of the sound and electronically encodes them. It sends the coded signal back to the transmitter [3], which sends the signal to the receiver/stimulator [4]. For the cochlear implant device (CI), the signal is sent along a wire to the electrode array implanted within the turns of the cochlea $(\mathbf{C o})$. The electrodes then stimulate the nerve endings in the cochlea, and the signal passes toward the brain via the auditory nerve (AN). For the auditory brainstem implant device (ABI), the signal is sent by the receiver/stimulator directly to the ABI electrode array, which is implanted within the lateral recess of the fourth ventricle, just below the cochlear nucleus $(\mathbf{C N})$.

information is then sent to the brain. The surgery is performed under general anaesthesia via a mastoidectomy approach (9). The first generation of cochlear implants consisted of a device with a single electrode (or channel). Technology has since evolved to systems that transmit more sound information through multiple electrodes. These multichannel cochlear implants can stimulate nerve fibers at different locations along the length of the cochlea to allow the user to hear many different pitches, which may provide better understanding of speech.

Of course, it is essential that a cochlear implant candidate have an intact acoustic nerve that is responsive to electrical stimulation, and it is this factor which, in most cases, makes cochlear implantation and acoustic tumor removal mutually exclusive. In most vestibular schwannoma excisions, the integrity of the cochlear nerve is sacrificed, thereby making later cochlear implantation impossible. This is especially true in cases of NF-2, where the lesion is known to invade the cochleovestibular nerve itself and grow within it, while unilateral acoustic neuromas (not associated with NF-2) tend instead to compress the nerve against the wall of the internal auditory canal (10). Cases have been reported, however, in which patients have undergone successful cochlear implantation ipsilateral to prior tumor excision where hearing preservation has been unsuccessful but the cochlear nerve left functionally intact (11). Ironically, of all acoustic tumor patients, it is those with NF-2 who are most likely to need some sort of neurostimulatory device to provide sound, since they often become totally deaf after the removal of their second lesion associated with the other cochlear nerve. In contrast, unilateral acoustic neuroma patients often have sufficient hearing in their non-tumor ear and therefore do not require the implant in the affected ear. New hope has been given to NF-2 
patients with the creation of the auditory ABI, which will be discussed later.

Even when the cochlear nerve is anatomically spared, it is often unresponsive to electrical stimulation once tumor excision has taken place. Therefore, even if all possible measures are taken to preserve the nerve during surgery, subsequent cochlear implantation may not be a feasible way of restoring sound to the patient. This is probably the greatest argument against simultaneous tumor excision and cochlear implantation: with the patient anesthetized, it is difficult to predict with any certainty whether or not the cochlear nerve has been preserved functionally, even if it has been preserved anatomically. Intraoperative electrically evoked auditory brainstem response testing may, however, aid in this decision process $(12,13)$.

While the functional integrity of the cochlear nerve is necessary for cochlear implantation, it is not the only issue which may render an acoustic tumor patient anatomically ineligible. It is thought that the neural element which is actually stimulated by an intracochlear electrode is the spiral ganglion cell, and it is therefore crucial that there exist an adequate population of spiral ganglion cells if cochlear implantation is to be successful (14-17). This is a very relevant concern in patients who have had their tumors removed via the translabyrinthine approach, because a transmastoid labyrinthectomy of this type is traumatic to the delicate structures of the inner ear. Chen et al. have reported, however, that a temporal bone which has undergone a transmastoid labyrinthectomy contains at least as many spiral ganglion cells eight years after labyrinthectomy as exist in the ear of a successfully implanted patient (14). In animals, Smith and Simmons have reported that only five to ten percent of the normal number of functional spiral ganglion cells are needed to produce electrically evoked auditory brainstem potentials (18). A related study has noted the presence of less than ten percent of the normal number of spiral ganglion cells in post-mortem studies of two successful cochlear implant users (19).

In addition to surgical damage which may be done to the cochlea, it is thought that the tumor's very presence may be damaging as well. This is due to the inherently parasitic nature of the schwannoma, as well as its existence as an obstacle to blood flow along the cochlear artery (R. Harrison, personal communication). If enough injury has been suffered by the neural cells of the cochlea because of the lack of oxygen this disruption of blood flow would cause, successful cochlear implantation may also not be possible.

\section{AUDITORY BRAINSTEM IMPLANT}

The management of NF-2 patients with bilateral acoustic neuromas from an auditory perspective has always been a challenge to physicians, since one must consider the size of the lesions (or the realistic chance of hearing preservation if such surgery were to be performed), the degree of hearing loss at diagnosis, and other factors, to determine the most appropriate course of action. While such management often extends the amount of time before the patient becomes deaf, the unfortunate reality is that patients will almost always be totally deafened after the removal of their second acoustic tumor.

The recent development of the auditory brainstem implant at the House Ear Institute (Los Angeles, California, USA) has given new hope to patients with NF-2 (Table 1). As its name suggests, the ABI is placed and acts directly on the brainstem, thereby bypassing all structures which lie peripheral to that point. This effectively avoids most of the problems associated with cochlear implantation in acoustic tumor patients since the health of the inner ear structures and the functional integrity of the auditory nerve become less relevant to successful prosthetic stimulation (20).

The ABI functions by electrically stimulating the cochlear nuclear complex, which consists of the dorsal and ventral cochlear nuclei (Figure 1). The ventral cochlear nucleus is the site at which the axons of the auditory nerve terminate. The device is not inserted on these structures directly, however, since such placement would lessen the distance and increase the likelihood of contact between the device and various other cranial nerves (particularly the facial (cranial nerve VII) and the glossopharyngeal (cranial nerve IX)) which is thought to increase the possibility of nonauditory sideeffects once stimulation begins (20). Instead, the ABI multielectrode prosthesis is inserted in the lateral recess of the fourth ventricle, which is adjacent to both cochlear nuclei. There is another advantage associated with this placement location as well: the cavity-like shape of the lateral recess aids in holding the electrode array in place, with the help of a small piece of fat which is packed into the meatus of the recess (20). This lessens the likelihood of a shift in the location of the device. As with the cochlear implant, a speech processor is worn on the patient's body and a transmission coil or plug, placed on the skin, transmits sound to the implant (20).

All patients who have been implanted with the ABI to date have undergone translabyrinthine tumor excision at the same time (22); the implant is inserted by this approach only, since it provides the surgical team with the greatest access to the specific sites which will be manoeuvred during the implantation procedure (23). The number of implantees to date is still relatively small (compared to the number of cochlear implantees) with 
roughly thirty individuals having thus far received the newly-developed multichannel ABI (23).

One concern often overlooked when dealing with patients with either NF-2 or unilateral acoustic neuroma is the effect that any implantation will have on the patient's ability to undergo subsequent magnetic resonance imaging (MRI). While undergoing an MRI scan, the patient is exposed to tremendous magnetic fields (24). Individuals who have been implanted with any sort of metallic device which may be displaced by the force of the magnetic field are usually forced to rely on the technique of computerized tomography (CT). This includes individuals with cochlear implants (25). In patients with unilateral acoustic neuroma, the inability to undergo MRI is a lesser consideration, since such individuals are less likely to require that imaging procedure. Patients with NF-2, however, are faced with a condition where they may develop various central nervous system lesions (including, perhaps, a contralateral acoustic neuroma) which are best treated with early diagnosis. This is less likely to be achieved if the patient is reliant on CT-scanning, which is significantly less accurate for smaller lesions. Fortunately, the ABI has been designed in such a way that it is not a contraindication to MRI. While some patients receive implants whose hardware includes a transcutaneous coil, most receive implants equipped with a percutaneous plug. The plug has several advantages over the coil, one being that the plug is made of non-ferrous materials which pose no threat to the patient should they later require MRI (20).

\section{FINAL COMMENTS}

Both the cochlear and auditory brainstem implants have been shown to aid significantly in providing sound to individuals suffering from total sensorineural hearing loss. It is somewhat difficult to directly compare the two based on previous studies, since both types of implants are constantly being updated. In general, however, it has been shown that the ABI, particularly the more recent multichannel ABI, provides levels of sound detection and discrimination which are similar to those provided by the original single channel cochlear implant (26). The two types of implants differ mainly in their ability to allow the patient to detect pitch, primarily because the ABI stimulates a region with no known tonotopic organization, whereas the multichannel cochlear implant takes advantage of the fact that the cochlea is tonotopically organized (26). While quite rare, some multichannel ABI patients have a limited ability to use the telephone $(22,27)$. It has even been reported that some ABI recipients demonstrate performance levels at certain tasks (such as sound-only sentence recognition) which are similar to those demonstrated by multichannel cochlear implant users (22).

Although they act by stimulating different levels of the auditory pathway, both cochlear and auditory brainstem implants can provide at least minimal acoustic stimulation to individuals who would otherwise be in a world devoid of sound. While it is assumed by many that cochlear implants are inappropriate for acoustic tumor patients (considering that the tumor and its surgical removal usually leave the cochlear nerve anatomically and functionally compromised), and that individuals with bilateral acoustic neurofibromatosis can benefit only from the $\mathrm{ABI}$, it is important that it be recognized that the issue is not that easily resolved. Although the vast majority of acoustic tumor patients truly are not cochlear implant candidates, making such an assumption at the outset without proper evaluation may deprive the individual (if he or she is not diagnosed with NF-2 and thus is not an ABI candidate) of a potentially useful prosthetic tool.

\section{ACKNOWLEDGMENTS}

The authors gratefully acknowledge Jaime Bederman for her editorial assistance in the preparation of this manuscript.

\section{REFERENCES}

1. Moffat DA, Hardy DG, Irving RM, et al. Referral patterns in vestibular schwannomas. Clinical Otolaryngology 20(1): 80-83; 1995.

2. Moffat DA, Baguley DM, Von Blumenthal H, et al. Sudden deafness in vestibular schwannoma. Journal of Laryngology and Otology 108(2): 116-119; 1994.

3. Consensus Development Panel. National Institutes of Health Consensus Development Conference Statement on Acoustic Neuroma, December 11-13, 1991. Archives of Neurology 51(2): 201-207; 1994.

4. Rouleau GA, Wertelecki W, Haines JL, et al. Genetic linkage of bilateral acoustic neurofibromatosis to a DNA marker on chromosome 22. Nature 329(6136): 246-248; 1987.

5. Shelton C. Surgical approaches for the removal of acoustic tumors. Seminars in Hearing 10(4): 334-340; 1989.

6. Slattery WH III, Brackmann D. Results of surgery following stereotactic irradiation for acoustic neuromas. American Journal of Otology 16(3): 315-319; 1995.

7. Deen HG, Ebersold MJ, Harner SG, et al. Conservative management of acoustic neuroma: an outcome study. Neurosurgery 39(2): 260-266; 1996.

8. Concensus Development Panel. National Institutes of Health Concensus Development Conference Statement on Cochlear Implants. Archives of Otolaryngology, Head and Neck Surgery 115(1): 31-36; 1989.

9. Cochlear Implant: A Device to Help the Deaf Hear. American Academy of Otolaryngology-Head and Neck Surgery, Inc. Patient Information Pamphlet (1990)

10. Hoffman RA, Kohan D, Cohen NL. Cochlear implants in the management of bilateral acoustic neuromas. American Journal of Otology 13(6): 525-528; 1992.

11. Briggs RJS, Popovic EA, Brackmann DE. Recent advances in 
the treatment of neurofibromatosis type II. Advances in Otolaryngology - Head and Neck Surgery 9: 227-245; 1995.

12. Nadol JB Jr, Chiong CM, Ojemann RG, et al. Preservation of hearing and facial nerve function in resection of acoustic Neuroma. Laryngoscope 102(10): 1153-1158; 1992.

13. Arriaga MA, Marks S. Simultaneous cochlear implantation and acoustic neuroma resection: Imaging Considerations, Technique, and Functional Outcome. Otolaryngology - Head and Neck Surgery 112(2): 325-328; 1995.

14. Chen DA, Linthicum FH Jr, Rizer FM. Cochlear histopathology in the labyrinthectomized ear: Implications for Cochlear Implantation. Laryngoscope 98(11): 1170-1172; 1988.

15. Belal A, Ylikoski J. Pathology as it relates to ear surgery, II: Labyrinthectomy. Journal of Laryngology and Otology 97(2): 1$10 ; 1983$.

16. Eddington DK, Dobelle WH, Brackmann DE, et al. Auditory prostheses research with multiple channel intracochlear stimulation in man. Annals of Otology, Rhinology, and Laryngology 87(Supplement 53): 1-39; 1978.

17. Zwolan TA, Shepard NT, Niparko JK. Labyrinthectomy with cochlear implantation. American Journal of Otology 14(3): 220-223; 1993.

18. Smith L, Simmons FB. Estimating Eighth Nerve Survival by Electrical Stimulation. Annals of Otology, Rhinology, and Laryngology 92(1 pt 1): 19-23; 1983.

19. Linthicum FH Jr, Fayad J, Otto S, Galey FR, House WF. Inner ear morphologic changes resulting from cochlear implantation. American Journal of Otology 12(Suppl): 8-10; 1991.

20. Brackmann DE, Hitselberger WE, Nelson RA, et al. Auditory brainstem implant: I. issues in surgical implantation. otolaryngology - Head and Neck Surgery 108(6): 624-633; 1993.

21. Laszig R, Sollmann WP, Marangos N, Charachon R, Ramsden R. Nucleus 20-channel and 21-channel auditory brain stem implants: first european experiences. Annals of Otology, Rhinology, and Laryngology 166(Suppl): 28-30; 1995.

22. Otto SR, Shannon RV, Brackmann DE, Hitselberger WE, Staller S, Menapace C. The Multichannel auditory brainstem implant: performance of 26 patients. Otolaryngology - Head and Neck Surgery (in press).

23. Otto SR, Brackmann DE, Staller S, Menapace CM. The multichannel auditory brainstem implant: 6-month coinvestigator results. Advances in Oto-Rhino-Laryngology 52(1): 1-7; 1997.

24. Portnoy WM, Matucci K. Cochlear implants as a contraindication to magnetic resonance imaging. Annals of Otology, Rhinology, and Laryngology 100(3): 195-197; 1991.

25. Hulka GF, Estrada JB, Pillsbury HC. Cochlear implantation in a patient after removal of an acoustic neuroma. The Implications of Magnetic Resonance Imaging with Gadolinium on Patient Management. Archives of Otolaryngology, Head and Neck Surgery 121(4): 465-468; 1995.

26. Shannon RV, Fayad J, Moore J, et al. Auditory brainstem implant: II. postsurgical issues and performance. Otolaryngology - Head and Neck Surgery 108(6): 634-642; 1993.

27. Kartush JM, Brackmann DE. Acoustic neuroma update. Otolaryngologic Clinics of North America 29(3): 377-392; 1996.

Neil L. Davis received his B.A. degree in Psychology from McGill University (Montreal, Quebec, Canada) in 1997. He is currently a research assistant in the Department of Otolaryngology at the Sir Mortimer B. Davis - Jewish General Hospital (Montreal, Quebec, Canada) and sits on the Board of Directors of the Acoustic Neuroma Association of Canada. He hopes to pursue a career in medicine. His project on hearing implants in acoustic neuroma patients was conducted as part of an independent study course in the Department of Psychology at McGill. 